\title{
An Immune-Inspired Approach for Unsupervised Texture Segmentation using Wavelet Packet Transform
}

\author{
Karinne S. Silva, Yuzo Iano \\ School of Electrical and Computer Engineering \\ State University of Campinas \\ Campinas, São Paulo, Brazil \\ \{karinne,yuzo\}@decom.fee.unicamp.br
}

\begin{abstract}
In this paper, it is described a new unsupervised approach based on wavelet packet transform for texture images segmentation. This transform is able to decompose an image not only from the low frequency parts, but also from the middle-high frequency parts, in which there is a certain amount of texture information. After the extraction of the features, a clustering is carried out, by using an immune-inspired algorithm called ARIA (Adaptive Radius Immune Algorithm), which is capable of preserving the density information of the data and determining how many different textures (clusters) are present in the image. The performance of our methodology is compared with other methods described in literature.
\end{abstract}

Keywords-texture analysis; texture segmentation; wavelet packet; ARIA.

\section{INTRODUCTION}

Textures contain important information for humans and are much used in the interpretation and analysis of various types of surfaces and objects. Besides, they can be defined as a set of spatial relations and arrangements of elements in a neighborhood.

Textural analysis is a difficult task in image processing. However, this is a fundamental step in many applications such as remote sensing, medical diagnosis, pattern recognition and surface inspections.

According to Livens [1] we can divide texture analysis methods into 4 segments:

- Statistics: In general, these methods achieve higher rates of discrimination than the structural methods and transforms. Examples of such methods are histogram analysis [2], co-occurrence matrix [3] and texture spectrum;

- Structurals: In these methods, the texture is defined as a composition of primitive patterns organized according to some rules. They have the advantage of producing a good description of the image, but are more useful to synthesis. An example of a tool for structural analysis is mathematical morphology [4];

- Models: Perform the analysis using mathematical models (stochastic and fractal). Some image parameters are extracted from the model and used as features to analyze the image. Its disadvantage is the computational complexity in the estimation of parameters [5];

- Transforms: In this case, the image is represented in a new space, for example the frequency/scale space, where the textural characteristics become more accessible. The main examples are the transform images: Fourier, Gabor [6] and Wavelets [7].

A large number of research works used to analyze texture image were proposed in the past [8], [9], [10]. But the traditional statistical approaches to texture analysis are restricted to the texture on a single scale.

Recent studies of human vision system suggest that the spatial/frequency representation, preserving global and local information, is adequate for quasi-periodic signals [11]. This has motivated new developments in multiresolution texture models. Currently, methods based on wavelet transform have received a lot of attention by providing analysis and characterization of the signal in different scales [7], [11], [12].

Coifman and Wickerhauser [13] generalized the wavelet basis function including a library of modulated waveform orthonormal bases called wavelet packets. This can decompose an image from the middle-high frequency parts. The wavelet packet decomposition is a generalization of the classical wavelet decomposition. The methodology described in this paper uses the wavelet packet decomposition to extract the characteristics of the pixels and perform the segmentation.

In this research, an immune-inspired algorithm called ARIA (Adaptive Radius Immune Algorithm) [14] was chosen to perform the clustering of the features of the textures. ARIA is capable of preserving the density information of the data and of automatically determining the number of clusters, which is fundamental for an unsupervised method.

The block diagram of the proposed method can be seen in Figure 1. Where $f(x, y)$ represents the original texture image, $W_{x, y}$ is the image wavelet packet transform, $t^{\prime}(x, y)$ represents the features, $t(x, y)$ represents the reduced features by principal component analysis, $L(x, y)$ is the result of ARIA clustering and $u(x, y)$ is the final segmentation image after passing by a median filter to remove small areas with wrong segmentation.

In the next section we will review the wavelet transform 


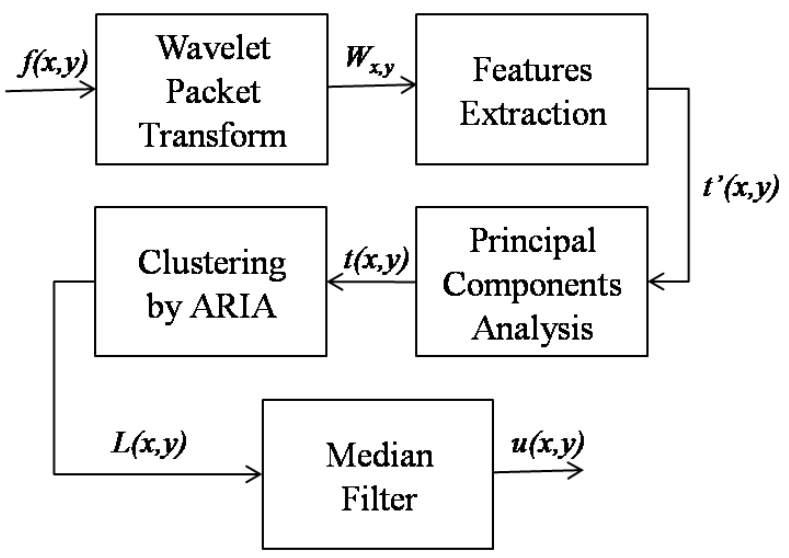

Figure 1. Block diagram of the proposed methodology

and the wavelet packet decomposition. Section III presents the method used to obtain the characteristics of the texture using the decomposition coefficients. The proposed methodology to image segmentation will be detailed in section IV. In section $\mathrm{V}$, the experimental results obtained with the proposed method can be found. Finally, section VI presents the conclusions.

\section{WAVELET PACKet TRANSForm}

\section{A. Wavelet Transform}

The main objective of the wavelet transform is to represent a signal as a superposition of basic orthogonal functions, called wavelets. These are obtained through dilation and translation of a single function called the mother wavelet, represented in 1

$$
\psi_{m, n}=2^{-m / 2} \psi\left(2^{-m} x-n\right)
$$

where $m$ and $n$ are integers [11]. The resulting wavelet decomposition coefficients of a signal $f(x)$ can be obtained by 2

$$
c_{m, n}=\int_{-\infty}^{\infty} f(x) \psi_{m, n}(x) d x
$$

and, the original signal can be obtained by 3

$$
f(x)=\sum_{m, n} c_{m, n} \psi_{m, n}(x) .
$$

The mother wavelet $\psi(x)$ is constructed from a scaling function $\phi(x)$ according to 4 and 5

$$
\begin{aligned}
& \psi(x)=\sqrt{2} \sum_{k} g(k) \phi(2 x-k) \\
& \phi(x)=\sqrt{2} \sum_{k} h(k) \phi(2 x-k)
\end{aligned}
$$

where

$$
g(k)=(-1)^{k} h(1-k) .
$$

The coefficients in 5 must comply with a number of restrictions for the set of basis wavelet functions to be orthonormal [11]. In the literature, several sets of coefficients can be found, so that some of the best known and utilized were proposed by Daubechies [15].

The coefficients $h(k)$, of the 10-tap and 16-tap Daubechies wavelet transform are listed in Table I. For 10tap Daubechies wavelet transform $h_{d}(k)=0$ if $k<0$ or $k>9$ and for 16-tap Daubechies wavelet transform $h_{d}(k)=0$ if $k<0$ or $k>15$.

Table I

WAVElEt TRANSFORM Filter COEFFICIENTS.

\begin{tabular}{lcc}
\hline & 10-tap Daubechies & 16-tap Daubechies \\
\hline$h(0)$ & 0.160102 & 0.054416 \\
$h(1)$ & 0.603829 & 0.312872 \\
$h(2)$ & 0.724308 & 0.675631 \\
$h(3)$ & 0.138428 & 0.585355 \\
$h(4)$ & -0.242294 & -0.015829 \\
$h(6)$ & 0.077571 & -0.000472 \\
$h(7)$ & -0.006241 & 0.128747 \\
$h(8)$ & -0.012580 & -0.017369 \\
$h(9)$ & 0.003335 & -0.044088 \\
$h(10)$ & & 0.013981 \\
$h(11)$ & & 0.008746 \\
$h(12)$ & & -0.004870 \\
$h(13)$ & & -0.000392 \\
$h(14)$ & & 0.000675 \\
$h(15)$ & & -0.000117 \\
\hline
\end{tabular}

We consider that $h(x)$ and $g(x)$ represent lowpass and highpass filters, respectively. They are used in wavelet transform decomposition to generate the approximation and details images. To obtain the various levels of decomposition, the approximation image is decomposed again in approximation images and detail images. The coefficients of approximation and details are in general used to obtain the characteristics for classification and segmentation of images.

\section{B. Wavelet Packet}

According to Zhang, He and Han [12], the decomposition of wavelet packet is a generalization of the classical wavelet decomposition. As in the Fourier method, any function $f(x) \in L^{2}(\Re)$ can be decomposed into wavelet packet functions.

The wavelet packet decomposition is capable of decomposing the signal in the middle-high frequency, not only in low and high frequency parts. This property is of fundamental importance for the characterization of texture, so that there is many textural information present in the intermediate frequency range [11].

The set of wavelet packet basis functions $\left\{W_{n}\right\}_{n=0}^{\infty}$ can be generated from a function $W_{0}$, as in 7 and 8

$$
W_{2 n}(x)=\sqrt{2} \sum_{k} h(k) W_{n}(2 x-k)
$$




$$
W_{2 n+1}(x)=\sqrt{2} \sum_{k} g(k) W_{n}(2 x-k)
$$

where $W_{0}$ and $W_{1}$ are similar to the scaling function and the mother wavelet, respectively. The library of wavelet packet bases can be defined as collection of orthonormal bases composed of functions of the form $W_{n}\left(2^{l} x-k\right)$, where $l, k \in Z, n \in N$. Each element of the library is determined by a subset of the indices: a scaling parameter $l$, a localization parameter $k$ and an oscillation parameter $n$ [11].

Figure 2 illustrates the tree-structured wavelet packet decomposition, where $S$ is the original signal, $D$ is the detail and $A$ represents the approximation image [12].

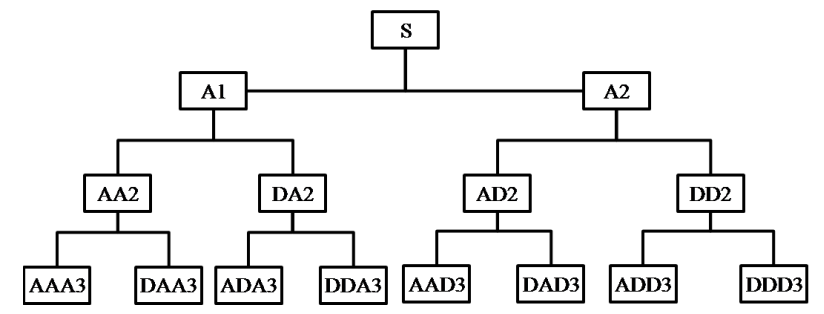

Figure 2. Tree-structured wavelets packet transform.

Since the most significant information of a texture often appears in middle frequency channels, further decomposition just in the lower frequency region, such as the traditional wavelet transform, may not be sufficient to carry out the segmentation. To illustrate this concept, Figure 3 shows a comparison of the wavelet transform and the wavelet packet decomposition of two textured images from Brodatz's album [16].

The key difference between this algorithm and the traditional wavelet transform is that decomposition is no longer simply applied to the low frenquency subsignals recursively. Instead, it is applied to the output of any filter $h_{L L}, h_{L H}$, $h_{H L}$ or $h_{H H}$ [11]. Thus, more detailed information can be obtained.

\section{Features Extraction using Wavelet Packet}

Initially, the images are decomposed by wavelet packet transform (level 1), presented in section II, and four subimages were obtained. Only the detail coefficients (vertical, horizontal and diagonal components) are used as features, and the information of the tone of gray in the low frequency sub-image is of no influence.

The energy of each component is calculated for each pixel according to 9

$$
E_{x, y}^{B}=f^{B}(x, y)^{2}
$$

where $B$ represents each of the sub-images and $f^{B}(x, y)$ is the value of the pixel $(x, y)$ in sub-image $B$.

For each pixel, three characteristics will be obtained, and before being used for segmentation of the image, they go

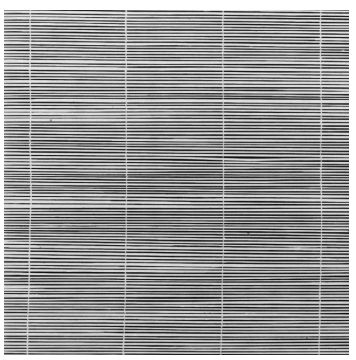

(a)

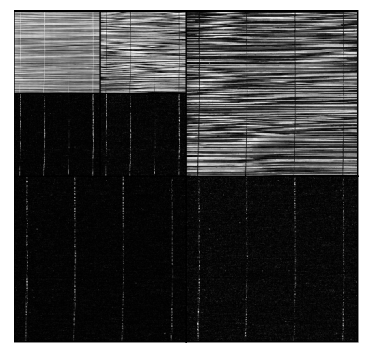

(c)

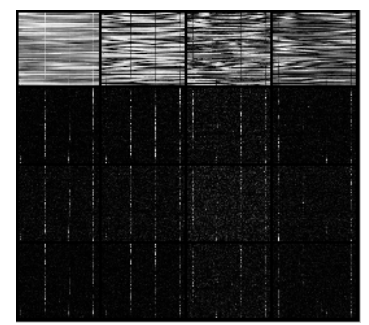

(e) (b)

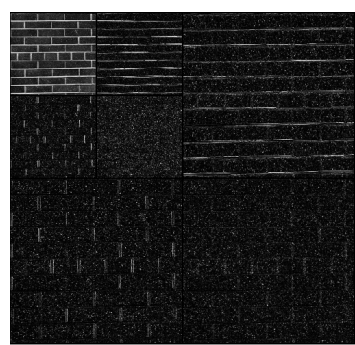

(d)

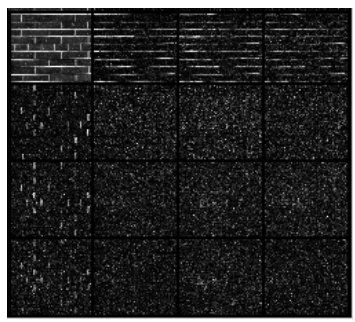

(f)

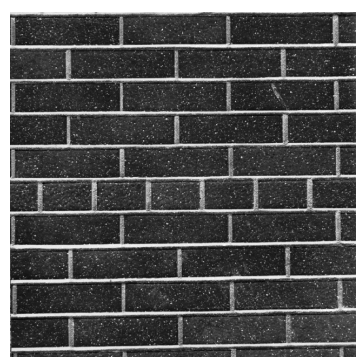

Figure 3. Wavelet transform (level 2) and wavelet packet transform (level 2) (a) Image D49 from Brodatz's album. (b) Image D26 from Brodatz's album. (c) Wavelet transform of image D49. (d) Wavelet transform of image D26. (e) Wavelet packet transform of image D49. (f) Wavelet packet transform of image D26.

to a process of standardization, positioning the values in the interval $[0,255]$.

In order to select only the most relevant characteristics, the Karhunen-Love transform was used, also known as principal components analysis [17], which reduces the number of features for only the most significant ones.

\section{Texture Segmentation}

After the extraction of the features for image segmentation, it is necessary to group the pixels that have the same textural characteristics. To perform this task, the algorithm ARIA (Adaptive Radius Immune Algorithm) was chosen [14].

The immune inspired algorithms are based on the natural immune systems, and in these algorithms the data are represented by antigens and the candidate solutions by antibodies. Some of these algorithms were proposed by de Castro and Von Zuben [18], [19] and de Castro and Timmis [20].

To obtain a better data representation, ARIA uses the properties of clonal expansion and network suppression 
along with the density of information of data. The algorithm is computationally fast and simple.

ARIA uses an adaptive suppression radius that is inversely proportional to the local density for each antibody's neighborhood, being capable of preserving the density information of the data. Thus, for high density data, the antibodies move closer to each other because of their small radius. In more sparse regions, the radius tend to be larger, so antibody distribution tends to be sparse too [14].

ARIA's procedure can be divided in three phases:

- Maturation: the antigens and the antibodies interact. In order to better fit the antigens, some antibodies suffer hypermutation.

- Clonal expansion: the most stimulated antibodies are cloned.

- Network suppression: if one antibody recognizes another, one of them is removed.

Algorithm 1 shows the pseudocode of ARIA, where $R$ is the radius of each antibody, $\mu$ is the mutation rate and decay is the constant used to decrease the mutation rate.

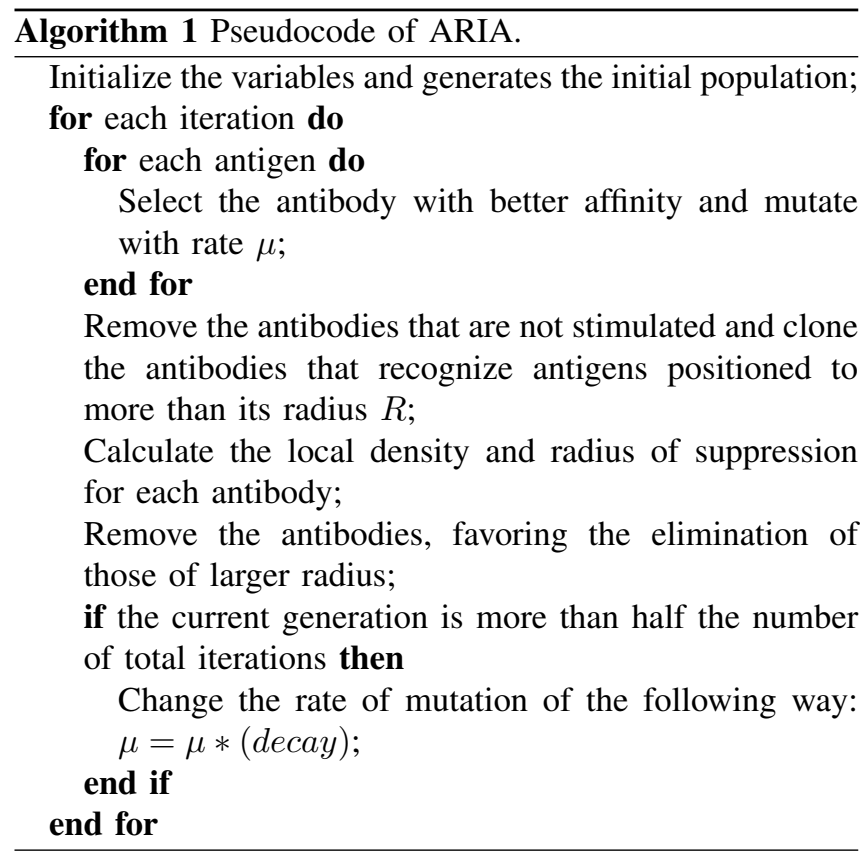

After convergence, it is necessary to define the network topology. To do that, the minimum spanning tree (MST) is used. The MST is interesting because it imposes a parsimonious structure to the network. Eliminating one of its edges always leads to subgraphs. This strategy is used to generate clusters [14], and each resulting subgraph corresponds to a cluster. To perform this task, a number of different criteria can be applied. In this work, the method chosen is: an edge is cut if it is longer than the median added $n$ times the variance of the length of its immediate neighbors.

Figure 4 shows some steps of an implementation of ARIA. Figure 4.(a) displays the network convergence, where 2000 points of data (antigens) are represented by 16 antibodies. In Figures 4.(b) and 4.(c) the MST's are shown.

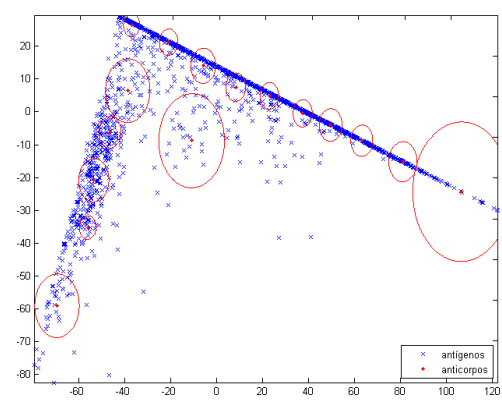

(a)

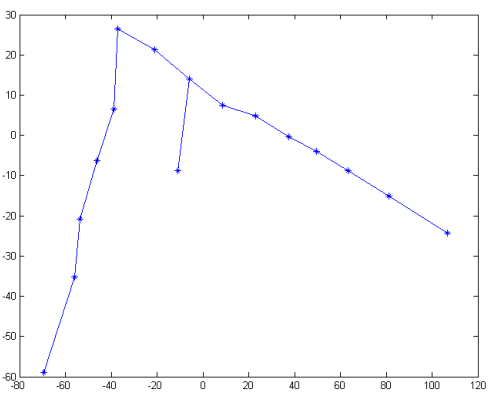

(b)

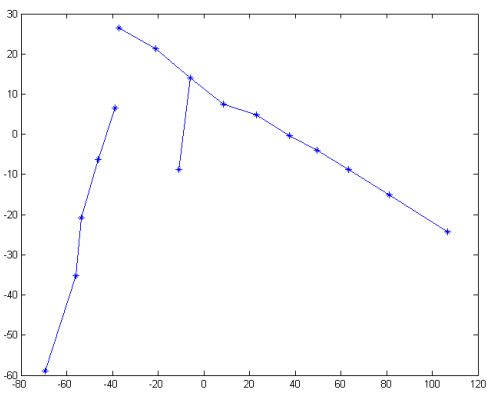

(c)

Figure 4. Execution of ARIA. (a) Network convergence. (b) MST. (c) Subgraphs formed by elimination of one edge of the MST.

After eliminating the inconsistent branches, each subgraph receives a label, which is passed to all antigens related with the subgraphs.

\section{EXPERIMENTAL RESULTS}

This section presents the performance of the proposed methodology. Initially, the texture image was decomposed using the wavelet packet transform. The features were obtained by the power of 2 of the decomposition components. After the features extraction, the principal component analysis was used, aiming to reduce the characteristics. The clustering was performed by ARIA algorithm. At last, the median filter of size $7 \times 7$ pixels was used to eliminate pixels that were erroneously classified.

Figure 5.(a) shows an image of size $157 \times 200$ pixels composed of the textures D49 (background) and D53 (inner 
pattern) of Brodatz's album [16]. In Figure 5.(b) the result obtained by Jenssen and Eltoft [21] is shown. The authors use the method ICA (Independent Component Analysis) to create bank of filters to texture segmentation. According to the authors, the ICA filters are similar to Gabor filters and are able to capture the inherent properties of textured images.

Figure 5.(c) presents the segmentation carried out by the methodology of Nunes and Conci [22] who proposed a new coefficient used in texture segmentation denominated CVE. This coefficient considers the information of spectral bands and allows to estimate the limits of areas too small or too large, locating the edges of the region of interest. It can be used for all types of texture because the rules to be identified are provided by the user and adapted to each situation.

Finally, Figure 5.(d) shows the segmentation obtained by the proposed method.

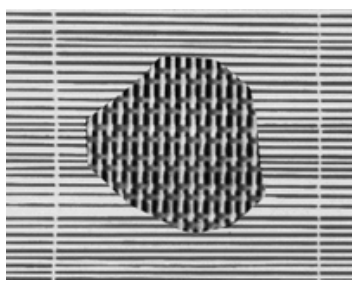

(a)

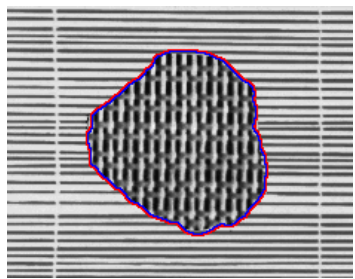

(c)

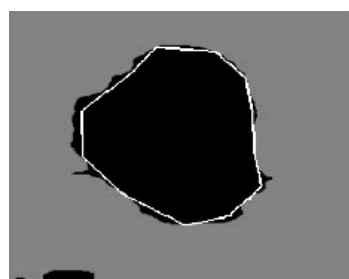

(b)

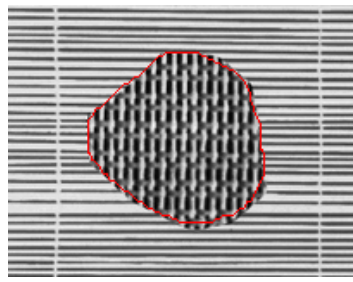

(d)
Figure 5. (a) Original image. (b) Segmentation obtained by Jenssen (black) and desired contour (white). (c) Result obtained by Nunes, in color. (d) Segmentation obtained by the proposed methodology.

It is observed that results obtained by the proposed method are better those presented by Jenssen and Eltoft [21]. However, a small drop in the quality of segmentation is seen in relation to that obtained by Nunes and Conci [22]. The latter uses a supervised method, where the user chooses samples of the textures to be used as training set.

Figure 6.(a) shows a 480x192 pixels monochromatic image, which represents the letters UCSB in the segments of the diagonal lines. This image is used as an example by the group JSEG, and it is also available on their web site [23]. Figure 6.(b) presents the result obtained by the group JSEG, Figure 6.(c) shows the result obtained with implementation of CVE [22] and Figure 6.(d) displays the result obtained with the proposed methodology. The contours were well located by the 3 methods.

Figure 7.(a) shows an image built using the texture D82 of Brodatz's album in the letters 'ABCDE'. The segmentation

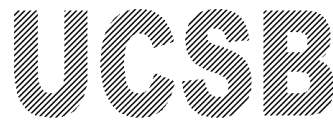

(a)

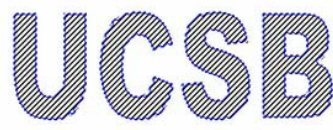

(c)

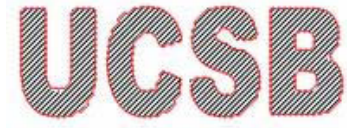

(b)

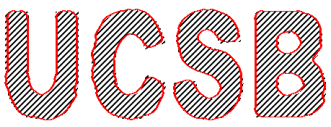

(d)
Figure 6. (a) Original image. (b) Segmentation obtained by JSEG. (c) Result obtained by Nunes. (d) Segmentation obtained by the proposed methodology.

obtained by the proposed methodology is displayed in Figure 7.(b). It can be seen that the contours are correctly localized with small errors in the inner part of letter 'A'.

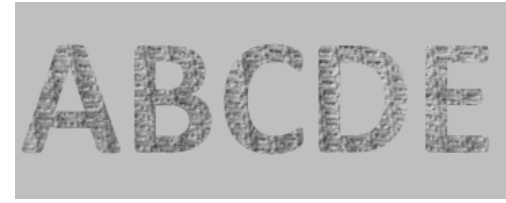

(a)

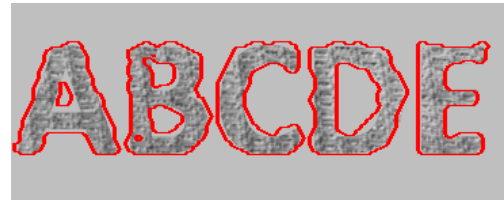

(b)

Figure 7. (a) Original image. (b) Segmentation obtained by the proposed methodology.

Figure 8.(a) presents an image composed of tree textures: D55 (top), D21 (bottom) and D24 (semicircle) of Brodatz's album. Figure 8.(b) shows the segmentation obtained by the proposed methodology. The contours of the tree textures are localized using only two features.

To emphasize the importance of a cluster algorithm capable of automatically determining the number of clusters, Figure 9 shows the results of segmentation of Figure 5.(a) obtained by k-means algorithm, well known in literature and widely used for clustering.

K-means perform a clustering based only on the data, the user has to determine the number of the clusters that are random initialized previously. This algorithm is subject to local minimums, but it is much used because of its simplicity of implementation.

It can be observed that, since the user has to set the numbers of clusters, different results can be obtained. In Figure 9.(a), the segmentation desired is not obtained and in Figure 9.(b), the segmentation would be obtained if the regions in black and gray were united. 


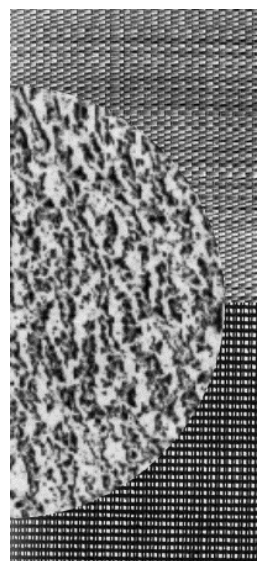

(a)

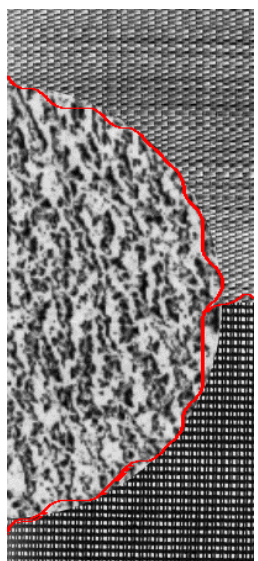

(b)
Figure 8. (a) Original image. (b) Segmentation obtained by the proposed methodology.

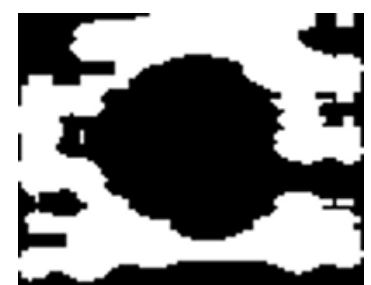

(a)

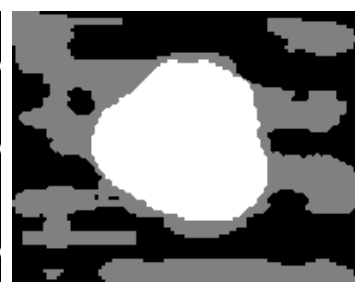

(b)
Figure 9. Segmentation by k-means. (a) Segmentation obtained by k-means for 2 clusters. (a) Result obtained by k-means for 3 clusters.

\section{CONCLUSIONS}

In this work, a new methodology for unsupervised segmentation of texture images was presented. The process can be divided in three principal steps: wavelet packet transform, features extraction and clustering.

The wavelet packet transform was chosen because it is capable of obtaining details of middle-high frequency, where the most significant information of a texture often appears.

To perform the clustering the ARIA algorithm was used. This immuno-inspired algorithm has the advantage of determining automatically the number of clusters, so the user might not have prior knowledge of the image. The experiments presented in this work showed that this is an important point in the segmentation result.

Experimental results presented prove the efficiency of our method. Several comparisons with other existing methods in literature were made. In some comparisons, the result obtained by our method was better, in others, the result was similar, but our method does not require the interference of the user.

\section{ACKNOWLEDGMENT}

The authors would like to thank the Brazilian funding agencies: FAPESP (Fundação de Amparo à Pesquisa do
Estado de São Paulo), FAEPEX (Fundo de Apoio ao Ensino, Pesquisa e Extensão), CNPq (Conselho Nacional de Desenvolvimento Científico e Tecnológico), CAPES (Coordenação de Aperfeiçoamento de Pessoal de Nível Superior) and Program CAPES/RH-TVD (Formação de Recursos Humanos em Televisão Digital) by supporting research developed in the Laboratório de Comunicações Visuais (LCV) of Unicamp.

\section{REFERENCES}

[1] S. Livens, "Image analysis for material characterization," Ph.D. dissertation, Antwerpen University, 1998.

[2] X. Liu and D. Wang, "Texture classification using spectral histograms," Image Processing, IEEE Transactions on, vol. 12, no. 6, pp. 661-670, Jun 2003.

[3] M. Hauta-Kasari, J. Parkkinen, T. Jaaskelainen, and R. Lenz, "Generalized co-occurrence matrix for multispectral texture analysis," vol. 2, Aug 1996, pp. 785-789.

[4] J. Sobus, B. Pourdeyhimi, B. Xu, and Y. Ulcay, "Evaluating loss of texture definition in carpets using mathematical morphology: Covariance," Textile Research Journal, vol. 62, no. 1, pp. 26-39, 1992.

[5] W. Nailon, S. McLaughlin, T. Spencer, M. Ramo, D. Salter, G. Sutherland, E. Fox, and W. McDicken, "Fractal texture analysis: an aid to tissue characterisation with intravascular ultrasound," vol. 2, Oct-2 Nov 1997, pp. 534-537 vol.2.

[6] A. Teuner, O. Pichler, and B. Hosticka, "Unsupervised texture segmentation of images using tuned matched gabor filters," Image Processing, IEEE Transactions on, vol. 4, no. 6, pp. 863-870, Jun 1995.

[7] M. Unser, "Texture classification and segmentation using wavelet frames," Image Processing, IEEE Transactions on, vol. 4, no. 11, pp. 1549-1560, Nov 1995.

[8] F. Cohen, Z. Fan, and M. Patel, "Classification of rotated and scaled textured images using gaussian markov random field models," Pattern Analysis and Machine Intelligence, IEEE Transactions on, vol. 13, no. 2, pp. 192-202, Feb 1991.

[9] N. Ahuja, "Mosaic models for textures," vol. 18, Dec. 1979, pp. 66-70.

[10] R. Haralick, "Statistical and structural approaches to texture," Proceedings of the IEEE, vol. 67, no. 5, pp. 786-804, May 1979.

[11] T. Chang and C.-C. Kuo, "Texture analysis and classification with tree-structured wavelet transform," Image Processing, IEEE Transactions on, vol. 2, no. 4, pp. 429-441, Oct 1993.

[12] Y. Zhang, X.-J. HE, and J.-H. Han, "Texture feature-based image classification using wavelet package transform," Lecture Notes in Computer Science, vol. 3644, pp. 165-173, Sep 2005.

[13] R. Coifman and M. Wickerhauser, "Entropy-based algorithms for best basis selection," Information Theory, IEEE Transactions on, vol. 38, no. 2, pp. 713-718, Mar 1992. 
[14] G. B. Bezerra, T. V. Barra, L. N. de Castro, and F. J. Von Zuben, "Adaptive radius immune algorithm for data clustering," Lecture Notes on Computer Science, vol. 3627, pp. 290-303, 2005.

[15] I. Daubechies, "Orthonormal bases of compactly supported wavelets," Communications on Pure and Applied Mathematics, vol. 41, no. 7, pp. 909-996, Oct 1988.

[16] T. Randen, "Brodatz textures," (http://www.ux.uis.no/ tranden/brodatz.html).

[17] S. Wold, K. Esbensen, and P. Geladi, "Principal component analysis," Chemometrics and Intelligent Laboratory Systems, vol. 2, no. 1-3, pp. 37-52, 1987.

[18] L. de Castro and F. Von Zuben, "Learning and optimization using the clonal selection principle," Evolutionary Computation, IEEE Transactions on, vol. 6, no. 3, pp. 239-251, fJun 2002.

[19] —-, Data Mining: A Heuristic Approach. Idea Group Publishing, 2002, ch. aiNet: An Artificial Immune Network for Data Analysis, pp. 231-259.

[20] L. de Castro and J. Timmis, "An artificial immune network for multimodal function optimization," vol. 1, May 2002, pp. 699-704.

[21] R. Jenssen and T. Eltoft, "Independent component analysis for texture segmentation," Pattern Recognition, vol. 36, no. 10, pp. 2301-2315, Oct 2003.

[22] E. O. Nunes and A. Conci, "Segmentação por textura e localização do contorno de regiões em imagens multibandas," IEEE Latin America Transactions, vol. 5, no. 3, pp. 185-192, 2007.

[23] "Jseg segmentation of color-texture regions in images and video," (http://vision.ece.ucsb.edu/segmentation/ jseg/jsegIllusionary.html). 